\title{
Magnetic Energy Storage and Conversion in Transient Solar Activity-Observations
}

\author{
Marcos E. MACHADO ${ }^{1}$ and Ronald L. MOORE ${ }^{2}$ \\ ${ }^{1}$ Department of Physics, The University of Alabama in Huntsville, Huntsville, Alabama 35899, U.S.A. \\ ${ }^{2}$ Space Science Laboratory, ES52, Marshall Space Flight Center, Alabama 35812, U.S.A.
}

(Received September 25, 1990; Revised December 25, 1990)

\begin{abstract}
The driver of transient solar activity is magnetic free energy stored in nonpotential configurations. This energy is released impulsively, within a period of the order of 10 to 1000 seconds, by an as yet not completely understood process or chain of processes. We review our current knowledge of the observed characteristics of energy buildup and release, and the limitations of our understanding. We conclude that the coordinated international research effort, FLARES 22, is necessary for an effective attack on this problem of great importance to astrophysics, space physics, and space exploration.
\end{abstract}

\section{Background}

Ever since the Skylab X-ray, XUV and EUV images became available, we have known that the entire outer solar atmosphere is largely a consequence of magnetic fields that are rooted in the photosphere and pervade the chromosphere and corona. These observations made quite clear that in order to understand solar atmospheric phenomena, as well as those in any similar star, we have to understands the causes and effects of magnetic activity (MOORE and RABIN, 1985; MACHADO and MOORE, 1986; PARKER, 1987).

The magnetic field in the solar atmosphere is built from flux loops that bubble up from below the photosphere. This flux is thought to be generated deep in the Sun's convective mantle by the dynamo action of global flow. The global patterns and evolution of the observed field then must reflect these flows. In contrast to this control of the field by the plasma at and below the surface, above the surface it is observed that the field strongly controls the structure (Fig. 1), dynamics and energy balance of the solar atmosphere. The field is apparently necessary for the very existence of the chromosphere, corona and solar wind, and it is obviously necessary for the occurrence of active transient phenomena like flares and filament eruptions, which are the prime subjects of this review.

In Section 2 we review pre-flare phenomena and observational evidence of magnetic free energy buildup. Section 3 deals with the observed characteristics of flare energy release.

\section{Pre-Flare Configuration and Energy Buildup}

Solar active phenomena are intimately related with evolving magnetic fields (see TANDBERG-HANSSEN and EMSLIE, 1988 and GAIZAUSKAS, 1989, for recent reviews). The 


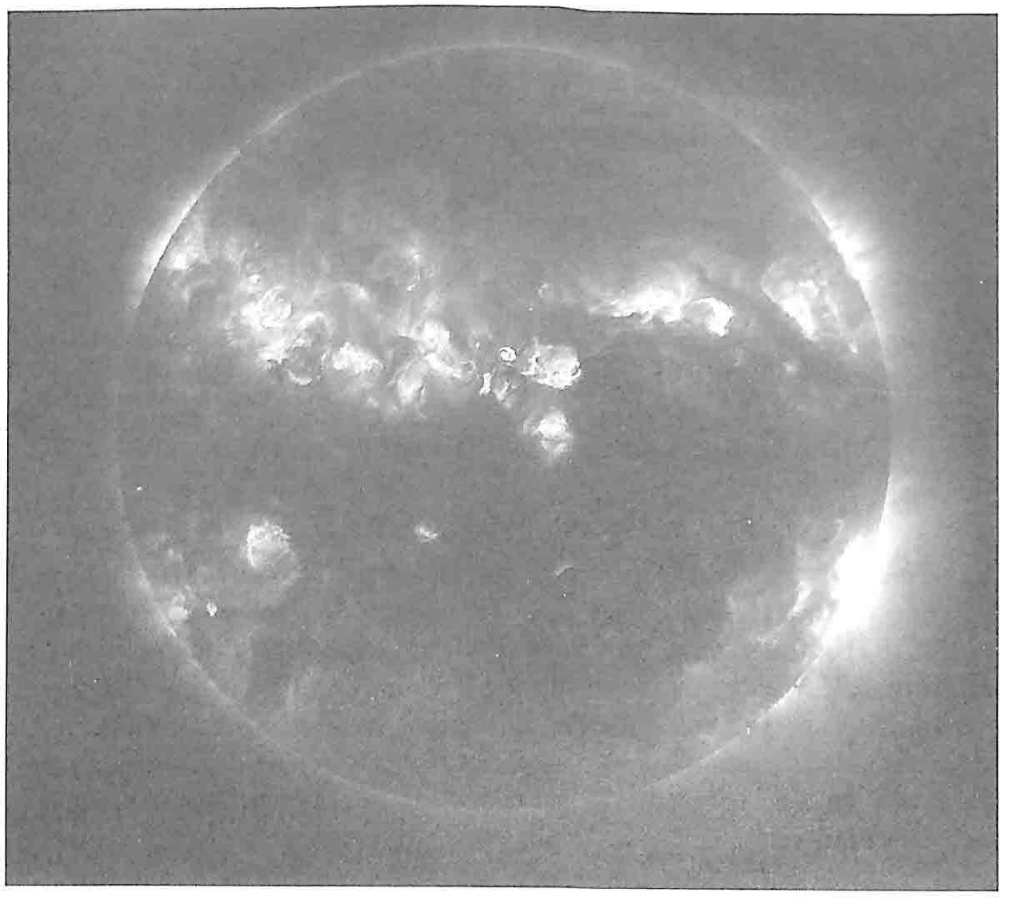

Fig. 1. High resolution X-ray image of the solar corona, showing magnetic loops filled with $10^{6} \mathrm{~K}$ plasma. The lengths of the observed loops range from several arcseconds $(1 "=725 \mathrm{~km}$ at Sun center) to several arcminutes. This high quality picture was obtained by the Normal Incidence X-ray Telescope (NIXT, photo credit IBM Watson Research Center and Smithsonian Astrophysical Observatory). Courtesy Dr. P. Martens.

source of energy of flares and eruptions is now universally accepted to be the magnetic free energy, which is stored in non-potential field configurations. However, after many years of study, the buildup of conditions that lead to irreversible transient activity remains as one of the most elusive and least understood aspects of flare physics.

On the other hand, with the advent of vector magnetic field observations like those obtained at the Marshall Space Flight Center (MSFC, see HAGYARD et al., 1982), quantitative measurements of the non-potential nature of the field became possible. The measurements have confirmed that flares are prone to occur in regions of high "observed magnetic shear". This quantitative estimate is obtained by comparing potential and observed field configurations. The line-of-sight component of the photospheric field can be used to calculate the structure of the potential field which is appropriate for such a configuration. This result can then be compared with measurements of the transverse component, to determine how much the observed field deviates from the potential. This comparison defines a so-called "shear angle", which is the difference between the direction of the transverse field component observed in the photosphere and the direction of the transverse component of the calculated potential field (Fig. 2). The value of the shear angle thus gives a measure of how much the field has been stressed. If the field is potential then the shear angle is $0^{\circ}$, while highly non-potential fields can lead to values up to $90^{\circ}$. 

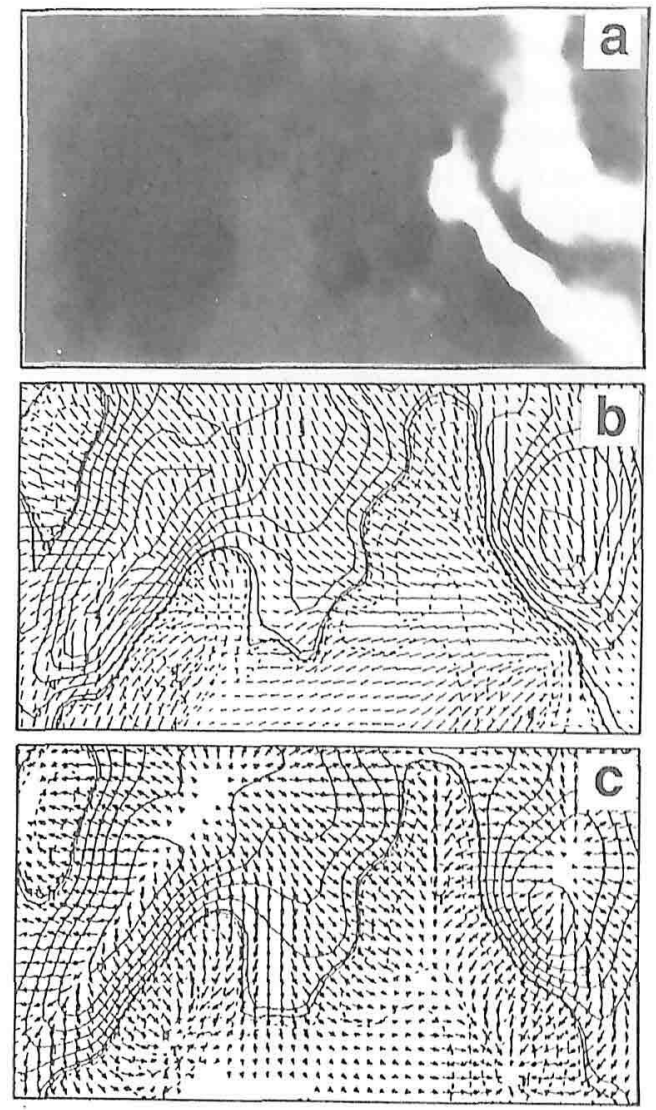

Fig. 2. Nonpotential fields in the region of the large flare of April 24, 1984. (a) H-alpha picture showing the bright flare ribbons that bracket the longitudinal magnetic neutral line. (b) Vector magnetogram obtained at MSFC, showing the longitudinal (full and dashed contours) and transverse (line segments) components. The strength and direction of the transverse component is indicated by the length and orientation of the line segments. (c) Display of the computed transverse component of the potential field in such a configuration. From a comparison between (b) and (c) we recognize that the field is highly nonpotential along the neutral line in the flare region. Note that other high shear places are observed (e.g., lower left-hand corner), but the flare occurred in the region of larger observed shear extent and field strength.

Although large shear seems to be a necessary condition for the occurrence of major events, it appears not to be sufficient. Many sites of strong shear may exist in an active region and not show strong activity (ATHAY et al., 1986; HAGYARD and RABIN, 1986; GAIZAUSKAS, 1989). More than 20 years ago, MORETON and SEVERNY (1968) pointed out that flaring is associated with strong field gradients. Allowing for the relatively low spatial resolution of their measurements, this implied that regions of strong oppositely directed fields are also necessary. This result was confirmed from observations obtained during the past solar maximum (see MACHADO et al., 1988 and TANDBERG-HANSSEN and EMSLIE, 1988, for updated lists of references). In agreement with this conclusion, the vector field 
measurements show that strong transverse fields, larger than 1000 Gauss, are usually observed at sites of strong flares. This is not surprising, since strong highly sheared fields contain more free energy than equally sheared weaker fields.

Even when both of the above conditions are met, this still does not mean that a large event is inevitable. In a recent study, HAGYARD (1990) has shown that a sufficient condition for strong flares to occur, in strong field and high shear areas, is for both to extend for at least $10000 \mathrm{~km}$ along the neutral line of the longitudinal field. Once again this result, albeit rather preliminary, has rather obvious implications: large volumes of stressed fields are needed to provide the energy to power a flare.

Having established that large areas of strong non-potential fields are necessary for production of strong flares, we now enter into the realm of the unresolved questions. The first is: how are sheared configurations formed?

Several responses have been given to this question, sometimes linking shear buildup to opposite phenomena like new flux emergence (ZIRIN, 1983) as well as submergence of already visible flux (HAGYARD et al., 1984; RABIN et al., 1985). The phenomenon of flux "cancellation" (MARTIN et al., 1985), which refers to the merging and disappearance of fine scale positive and negative polariries, has also been linked to the formation of shear. VAN BALLEGOOIJEN and MARTENS (1989), demonstrate that cancellation can be linked to flux removal by a combination of reconnection and submergence due to magnetic tension. They show that such a process may lead to the production of helical field structures which are capable of supporting prominences. Futher flux cancellation may then lead to the prominence eruption, which often accompanies the largest flare events and many coronal mass ejections (CMEs, see HILDNER and KAHLER in these proceedings).

Indirect evidence of shear buildup can be obtained from measurements of spot motions (GESZTELYI and KALMAN, 1986; HAGYARD and RABIN, 1986), as well as photospheric velocity field measurements which show vortical motions in concentrated field regions (MARTRES et al., 1982; MARTRES, 1989). A good way to look for shear buildup is in proxy indicators of field lines, like the $\mathrm{H}$-alpha fibrils seen at chromospheric levels. This technique was used several years ago by TANAKA and NAKAGAWA (1973), and has been recently reviewed by KUROKAWA (1989). Figure 3 shows an example of a highly sheared structure in a deltaspot configuration. Such a configuration, of opposite polarity spots in close contact, has been known for many years to be a productive site for strong flares (SVESTKA, 1976). In fact, adding the severe observed shear, the delta-spot meets all the requirements for flare production mentioned above.

In spite of the use of so many techniques, results linking the buildup of shear with flares and other transient activity remain quite conflictive and, as noted, sometimes contradictory. All sorts of dynamic processes have been shown to work in particular cases, and to fail in others, with the role of flux emergence as a clear example. Thought for many years to be the prime flare trigger candidate (PRIEST, 1981 and references therein), it is now widely accepted that new flux emergence does not have a direct correlation with flaring. It is true that many flares occur in regions of emerging flux, but it is also true that many episodes of flux emergence occur in the lifetime of an active region, without causing flares. It is possible, that the flare production by flux emergence may depend on the ambient field encountered by the emerging flux and/or the characteristics of the emerging flux itself. Emergence of new flux in a region "loaded" with magnetic free energy may trigger its instability. Conversely, the new flux may emerge loaded and release its energy when it interacts with the ambient field. Figure 4 shows a case in which magnetic flux apparently 


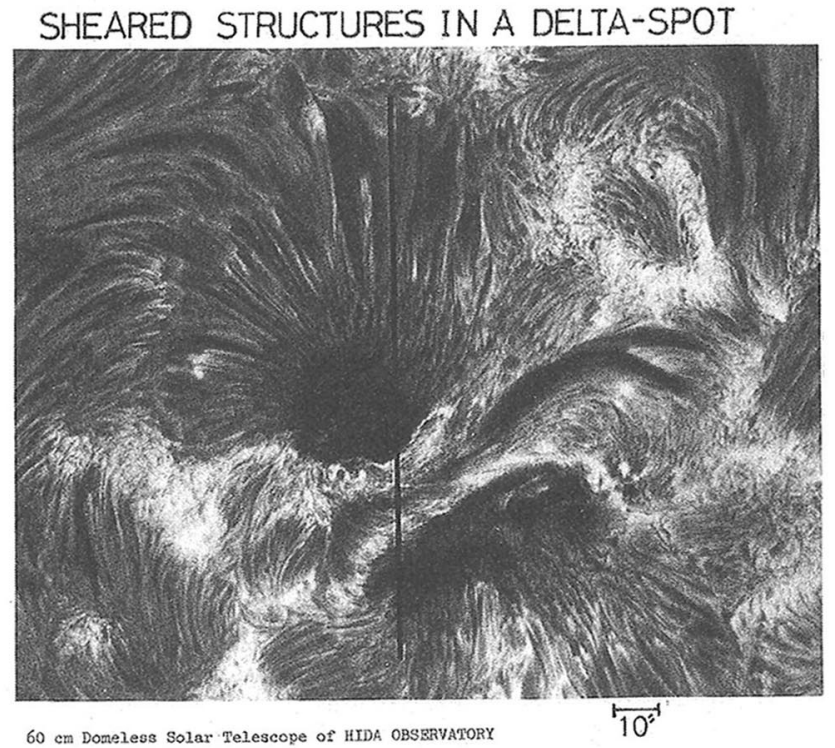

Fig. 3. H-alpha observation of a highly sheared configuration in a delta-spot region. The thin filaments between the two opposite polarity spots run almost parallel to the magnetic neutral line, delineated by a dark filament (Photo, courtesy Dr. H. Kurokawa, Hida Observatory).

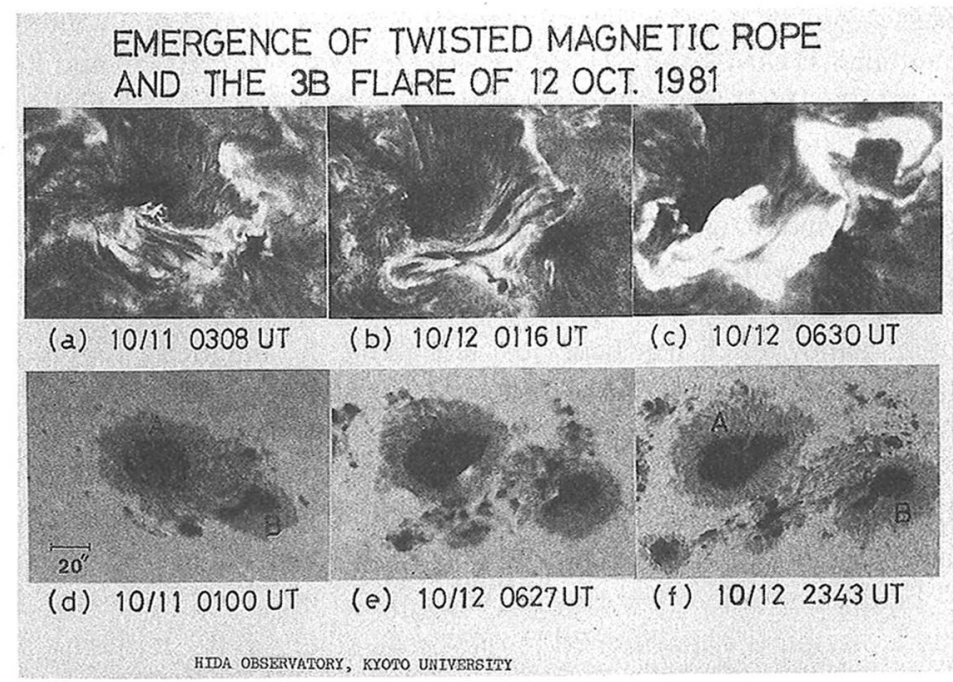

Fig. 4. Development of magnetic shear preceding a large flare on October 12, 1981. On October 11, an arch filament system is seen in H-alpha (a) and penumbral filaments in white light (d), connecting the two main spots (A and B) in a nearly potential configuration. By October 12 (b and e), newly appeared $\mathrm{H}$-alpha emerging loops are seen to run along the polarity inversion line, in a strongly sheared configuration between the spots. This led to the large flare shown in (c). Photo, courtesy Dr. H. Kurokawa, Hida Observatory. 
contained free energy when it emerged, and led to a large flare. MCCLYMONT and FISHER (1989), argue in favor of such subphotospheric storage as the most efficient mechanism for the energy buildup of large events.

A second, basic, question is: why and when is the magnetic free energy released? The next section deals with the many alternatives that have been proposed.

\section{Energy Release Characteristics}

Any flare, big or small, is found to sit in the core of a magnetic bipole, straddling the polarity inversion line (SVESTKA, 1976; MACHADO et al., 1988). In many active regions, the main inversion line of the overall bipole is traced by a dark filament, i.e. a prominence seen in projection onto the disk. Figures 3 and 4 show examples of this observational property. These filaments often show a twisted structure and fine scale strands that run nearly parallel to the neutral line, indication that they lie, as noted in Section 2, in strongly sheared fields.

Most major solar flares are accompanied by the preflare activation and subsequent eruption of a filament (MARTIN, 1980). The activation, which usually occurs within the hour preceding the flare, is characterized by a darkening, enhanced mass motions and outward expansion of the filament. Thesephenomena lead to the irreversible eruption in a continuous process. The start of the eruption is driven by the expansion of the sheared field, which is followed shortly thereafter by the rapid energization of plasma that characterizes the impulsive phase of the flare (TANAKA, 1987; KAHLER et al., 1988). Therefore, observations indicate that it is the flare that is driven by the eruption, rather than the field eruption being driven by a pressure pulse due to the flare energy release.

These observations then led to the concept of "critical shear", as a candidate for flare trigger. Many authors (BARNES and STURROCK, 1972; LOW, 1977; HOOD and PRIEST, 1980; BIRN and SCHINDLER, 1981), have advocated the idea that during the buildup of magnetic shear a critical value, beyond which the system losses equilibrium, must exist. This critical point can then be interpreted as a threshold for eruption and flare onset. Although attractive from a theoretical standpoint, and in spite of the clear correlation between flares and observed high shear angle, there is still no compelling observational proof of the existence of a critical shear value as a flare trigger.

On the other hand, much has been learned about the magnetic topology of flaring regions from recent multiwavelength observations. Analyses of SMM X-ray images (MACHADO et al., 1988), radio images of centimeter and meter wave sources (KUNDU, 1986; PICK et al., 1990; WILLSON et al., 1990), as well as H-alpha (TANG, 1985; KUROKAWA, 1989), have shown that flares usually encompass multiple magnetic bipoles within an active region. The basic structure of a flare seems to consist, in at least a great number of cases, of an "initiating" bipole and one or more adjacent bipoles impacted against it. During flare onset, the X-ray emission is concentrated but not seen solely in the initiating bipole. Radio observations show flare associated phenomena at different sites, confirming that already at this stage the flare byproducts have access to various magnetic structures. The impulsive phase of the flare is characterized by the rapid increase of emission, at all wavelengths but most notably in hard and soft X-rays, over the initiating bipole as well as in those impacted against it.

These results seem to indicate that the interaction of magnetic loops, as proposed in many current sheet models (see e.g. PRIEST, 1981, for a review), is an essential ingredient in the trigger of the high-power energy release in flares. However, some additional 
characteristics add to the simple picture of energy being released in a current sheet at the interface between two loops, first described by SWEET (1958).

The morphology and time evolution of hard and soft X-ray emission, show that the bulk of the flare energy is released, among those involved, within one of the participating bipoles. Typically, it is the bipole where vector magnetic field observations show the largest degree of stress as defined above, i.e. combination of high shear angle, field strength and shear area. The suggestion is, then, that although the role of current sheets between bipoles may serve, and perhaps be essential, for the trigger of flares, most of the time integrated energy output is drawn from the internal reservoir of magnetic free energy stored in individual bipoles. The most extreme example is given by cases in which one of the bipoles is the site of a filament eruption and CME. As noted by MACHADO et al. (1988), only a picture of widely varied amounts of energy storage and release in different magnetic structures, can explain the morphological, temporal and energetic properties of events ranging from compact brightenings to flares that may encompass entire active regions.

Therefore, it is clear that the time has come to consider, both observationally and theoretically, more realistic situations than the single loop or single arcade treated in the past. It is also clear that the characteristics of flares, as well as ejecta like CMEs, not only relate to local conditions but are also influenced by the global background configuration of the active region. HENOUX and SOMOV (1987) have shown how motions in an active region can create a complicated network of currents, along magnetic flux cells which interact at the separator (see also LOW and WOLFSON, 1988; GAIZAUSKAS, 1990 and MARTENS et al., 1990). Reconnection at the separator may induce current dissipation in individual bipolar features, leading to widespread flares of varied characteristics.

\section{Conclusions}

The lesson to be learned from the above discussion is that flares and other forms of magnetic activity are complex phenomena. We have seen the value of combining different observational techniques to define the characteristics of the preflare configuration. These are now quite well established, but we still lack information and physical understanding on how and why energy buildup takes place. Many key questions also remain concerning the mechanisms of energy release and dissipation. We see simplified flare concepts fade away, being replaced by another in which current-carrying loop systems within complex magnetic topologies interact, in an as yet imperfectly understood way, to release stored free energy in the form of heating, particle acceleration and mass motions. This lack of understanding is reflected in our still poor capabilities to predict solar activity and its consequences in the interplanetary medium and near Earth environment, an issue of substantial practical value in an era of space exploration.

The complexity of the solar activity phenomena is such, that it demands a concerted effort in observations from both the ground and space, as well as theoretical studies encompassing many branches of physical research. It is thus clear that the degree of effort demanded, far exceeds the individual capabilities of individual research groups. Only an organized worldwide effort can further our understanding of solar activity. For such a purpose, an international program of FLAre RESearch at the maximum of solar cycle 22, the FLARES 22 Project, has been implemented within the overall framework of SCOSTEP's Solar-Terrestrial Energy Program. The scientific aim of FLARES 22 is to provide, through coordinated observational campaigns and theoretical studies, a basic physical understanding of 
the synergistic interplay between solar magnetic fields and plasmas, the conversion of magnetic free energy, and its transport and dissipation. Further details about the scope and organization of FLARES 22 can be found in MACHADO and PICK (1988).

This review should have been written by Dr. Katsuo Tanaka, who passed away in January 1990. Katsuo's untimely death is a great loss for the international solar physics community. This article is dedicated to his memory.

\section{REFERENCES}

Athay, R. G., H. P. Jones, and H. Zirin, Astrophys. J., 303, 877, 1986.

Barnes, C. W. and . P. A. Sturrock, Astrophys. J., 174, 659, 1972.

BIRN, J. and K. SchINDLER, in Solar Flare Magnetohydrodynamics, edited by E. R. Priest, p. 337, 1981.

Gaizauskas, V., Solar Phys., 121, 135, 1989.

Gaizauskas, V., in Physics of Magnetic Flux Ropes, edited by C. T. Russell, E. R. Priest, and L. C. Lee, Geophysical Monographs 58, p. 331, 1990.

Gesztelyi, L. and B. Kalman, Adv. Space Res., 6, No. 6, 29, 1986.

Hagyard, M. J., Mem. Soc. Astron. Italiana, in press, 1990.

Hagyard, M. J., N. P. Cumings, E. A. West, and J. E. Smith, Solar Phys., 80, 33, 1982.

Hagyard, M. J., R. L. Moore, and A. G. Emslie, Adv. Space Res., 4, No. 7, 71, 1984.

Hagyard, M. J. and D. Rabin, Adv. Space Res., 6, No. 6, 7, 1986.

Henoux, J.-C. and B. V. Somov, Astron. Astrophys., 185, 306, 1987.

Hood, A. W. and E. R. Priest, Solar Phys., 66, 113, 1980.

Kundu, M., Adv. Space Res., 6, No. 6, 53, 1986.

KuroKawa, H., Space Sci. Reviews, 51, 49, 1989.

Low, B. C., Astrophys. J., 217, 988, 1977.

Low, B. C. and R. Wolfson, Astrophys. J., 324, 547, 1988.

Machado, M. E. and R. L. Moore, Adv. Space Res., 6, No. 6, 217, 1986

Machado, M. E. and M. PICK, Adv. Space Res., 8, No. 11, 121, 1988.

Machado, M. E., R. L. Moore, A. M. Hernandez, M. G. Rovira, M. J. Hagyard, and J. B. Smith, Jr., Astrophys. J., 326, 425, 1988.

Martens, P. C. H., L. Golub, and M. Herant, in Proceedings of the Workshop on Plasma Phenomena in the Solar Atmosphere, Cargese, 1990 (in press).

Martin, S. F., Solar Phys., 68, 217, 1980.

Martin, S. F., S. H. B. Livi, and J. Wang, Australian J. Phys., 38, 929, 1985.

Martres, M. J., Solar Phys., 119, 357, 1989.

Martres, M. J., J. Rayrole, M. Semel, I. Soru-Escaut, K. Tanaka, M. Makita, and F. Moriyama, Publ. Astron. Soc. Japan, 34, 299, 1982.

McClymont, A. N. and G. H. Fisher, in Solar System Plasma Physics, Geophysical Monograph 54, American Geophysical Union: Washington D.C., p. 219, 1989.

Moreton, G. E. and A. B. Severny, Solar Phys., 3, 282, 1968.

Moore, R. L. and D. Rabin, Ann. Rev. Astron. Astrophys., 23, 239, 1985.

PARKer, E. N., Physics Today, 40(7), 36, 1987.

PICK, M., K.-L. KLeIN, and G. Trottet, Astrophys. J. Suppl., 73, 165, 1990.

Priest, E. R. (ed.), Solar Flare Magnetohydrodynamics, Gordon and Breach, 1981.

Rabin, D., R. L. Moore, and M. J. Hagyard, Astrophys. J., 287, 404, 1984.

StUrrock, P. A. (ed.), Solar Flares-A Monograph from Skylab Workshop II, Colorado University Press, 1980.

SvestKa, Z., Solar Flares, D. Reidel Publ. Co., 1976.

Sweet, P. A., Nuovo Cimento Suppl., 8, Ser. X, 188, 1958.

TANAKa, K., Publ. Astron. Soc. Japan, 39, 1, 1987.

TANaka, K. and Y. Nakagawa, Solar Phys., 33, 187, 1973.

Tandberg-Hanssen, E. and A. G. Emslie, The Physics of Solar Flares, Cambridge University Press, 1989.

TANG, F., Solar Phys., 102, 131, 1985. 
van Ballegooijen, A. A. and P. C. H. Martens, Astrophys. J., 343, 971, 1989.

Willson, R. F., K.-L. Klein, A. Kerdraon, K. R. Lang, and G. Trottet, Astrophys, J., 357, 662, 1990. ZIRIN, H., Astrophys. J., 274, 900, 1983. 